\title{
CELESTINA DE FERNANDO DE ROJAS: DOCUMENTO BIBLIOGRÁFICO (decimoctavo suplemento)
}

\author{
Joseph T. Snow \\ Randal Garza \\ Michigan State University
}

[Seguimos las huellas de la celestinesca mundial, prolongando en estos suplementos la bibliografía - desde hace tiempo agotada que publicó la Hispanic Seminary (Madison, Wisconsin) en 1985. Se agradece, para materiales reseñados en este suplemento, a los siguientes colegas: Justo Alarcón de la Biblioteca Nacional de Chile, Félix Carrasco, Hensley C. Woodbridge, Víctor Infantes y Marcial Rubio, John Beusterian, Emma Scoles, Georgina Olivetto, y Harry Vélez Quiñones. Ed.]

670. BELLVER, Joaquín Aguirre. Los secretos de La Celestina. Madrid: Jaguibel, 1994.

La teoría es que hubo un borrador en verso de la obra antes de la forma en prosa que nos desconcierta tanto. Gran parte de este libro es una muestra de dicho borrador. Cree haber desentrañado el problema de autoría (Rojas como único autor) $y$ el significado en dos niveles (el narrativo, el alusivo).

671. BIOY CASARES, Adolfo. "La Celestina," en La otra aventura. Buenos Aires: Galerna, 1968. 7-21. 
Un prólogo a una edición de la TCM publicada por la casa Estrada, este comentario--además de situar a Rojas y su obra en un contexto literario--presenta algunos problemas básicos de Celestina: la sicología que motiva los personajes, el carácter general de la prosa y la originalidad de la Tragicomedia.

672. CARO BAROJA, Julio. Las brujas y su mundo (Madrid: Revista de Occidente, 1961), pp. 151-153.

Más que por la literatura latina, es por la realidad urbana castellana que Rojas llegó a conocer el tipo celestinesco que luego elaboró en $L C$.

673. "La Magia en Castilla durante los siglos XVI y XVII," en su Algunos mitos españoles y otros ensayos. (Madrid: Editora Nacional, 1944), pp. 185-303.

Celestina (passim) conectada con prácticas reales de la época, reflejadas aquí en un potpourrí de textos y documentos relevantes.

674. "Magia, sexo y estatuto social (el arquetipo celestinesco)," en su Vidas mágicas e Inquisición (Madrid: Taurus, 1967): I, 107134.

Representaciones de figuras de hechiceras (la lección magistral es la de Rojas en el aucto VII de su Celestina) Gil Vicente, Sancho de Muñón (Tragedia de Lisandro y Roselia), Sebastián Fernández (Tragedia Policiana), Alonso de Villegas (La Lena), Juan Navarro de Espinosa (entremés titulado La Celestina), Cervantes (El coloquio de los perros) Lope de Vega (El capellán de la virgen; la Dorotea), Gonzalo de Céspedes y Meneses, Juan de Zabaleta, Agustín de Salazar y Torres, Antonio de Zamora y J.E. Hartzenbusch. Matizaciones literarias e históricas acompañan esta excursión hechiceril.

675. CRUZ, Anne J. "Sexual enclosure, textual escape: the Pícara as Prostitute in the Spanish Female Picaresque Novel," en Seeking the Woman in Late Medieval and Renaissance Writings, eds. Sheila Fisher and Janet E. Halley. Knoxville, University of Tennessee, 1989. pp. 135-159. 
El origen de la (sexualmente) bien diferenciada 'picaresca femenina' se halla en el mundo bajo retratado por Fernando de Rojas: el mundo de la prostitución. Ese mundo florece cuando se mantiene distinciones entre 'buenas' y 'malas' mujeres. El mundo celestinesco colorea a los ambientes de Lozana andaluza, La pícara Justina, y La hija de Celestina.

676. DAVIES, Gareth A. "Destellos de un sub-texto literario: la rememoración del pasado en la Celestina," en Leeds Iberian Papers, Leeds Papers on Hispanic Drama 1991, ed. M.A. Rees (Leeds: Trinity \& All Saints College, 1991): 9-41.

Con una lectura cuidada de las referencias textuales al pasado dentro del texto de Celestina, el autor construye un contraste vital entre una sociedad rememorada y caracterizada por la tolerancia y fluidez social (un 'entonces') y una sociedad mucho menos marcada por esos antiguos valores (un 'ahora'). Especula sobre esta relación ficcional y se pregunta si estamos ante una rememoración personal (de parte de ambos autores) de los cambios efectuados por la aparición en España del Santo Oficio. Sería un sub-texto que refleja un conflicto más en la serie de cosas conflictivas detalladas en el 'Prólogo': el conflicto de épocas distintas (en este caso, épocas sucesivas).

677. DE VRIES, Henk. "¿Quién es la Lozana?" Celestinesca 18.1 (primavera 1994): 51-73.

Es un análisis del Retrato de la Lozana Andaluza basado en los personajes, sus números, extensión de sus intervenciones (con gráficos ilustrativos). Los años representados en la ficción, los nexos entre Lozana y Celestina, y las alusiones numéricas en el texto de Lozana al de Celestina son otros temas tratados. Inclúyese un apéndice alfabético de los personajes que hablan en Lozana.

678. EDWARDS, Jorge. "La Celestina, un Fausto con faldas." La Segunda (Santiago de Chile) (16 de octubre 1992), p. 8.

En los tiempos de Colón, Rojas es un precursor de Goethe. Observaciones al personaje de Celestina. 
679. FRADEJAS LEBRERO, José. "Cazar aves con lumbre (más antiguo aún)." Celestinesca 18.1 (primavera 1994): 75-77.

El 'cazar con boyezuelo' parece ser más antiguo (y oriental) de lo hasta ahora se ha comentado en la serie de artículos sobre el estratagema mencionado por Pármeno (acto XI). Ahora se cita la Historia natural de Plinio el Viejo (y su traductor español, Francisco Hernández, finales del s. XVI).

680. DE LA FUENTE, Darío. "Fernando de Rojas." Líder provincial (San Antonio, Chile) (18 abril 1991), pp. 3 y 5.

Una breve noticia general que incluye una de las pocas noticias de la ópera de Felipe Pedrell que se hizo a base de Celestina.

681. "La Celestina." El Espectador (San Antonio, Chile), (2 agosto 1991), p. 2.

Breve noticia de su contenido y sus méritos como creación de lenguaje.

682. GARCIA, Michel. "Consideraciones sobre 'Celestina' de Palacio." Celestinesca 18.1 (primavera 1994): 3-16.

Sugiere maneras distintas por medio de las cuales uno puede valorizar el fragmento celestinesco del manuscrito Palacio 1520. Justifica cómo las variantes estilísticas, entre el MS y otras versiones tempranas en la tradición textual, arrojan luz sobre las innovaciones de Rojas como (segundo) autor.

683. GARNEAU, Michel. "Célestine là-bas près des tanneries au bord de la rivière, d'après Fernando de Rojas." Traducción y adaptación de Michel Garneau. Montréal: ULB Editeur, 1991. Rústica, $168 \mathrm{pp}$. Foto en colores de la capa de Robert Etcheverry. Con once fotos (blanco y negro) que acompañan el texto.

Un arreglo dramático con 13 actores (falta Crito) en 21 "scènes." Comienza con el lamento de Pleberio y luego sigue el orden del original, incorporando buena parte del texto escrito. Estreno en el estudio del Centre Nationale des Arts à 
Ottawa (Canadá) el 16 de noviembre de 1990, dirigido por Jean Asselin.

684. GASCóN Vera, Elena. "La puta vieja filosofía: Boecio como subtexto de la Celestina," en su Un mito nuevo: La mujer como sujeto/objeto literario (Madrid: Ed. Pliegos, 1992), pp. 223-231.

Comenta la intertextualidad entre Celestina y La consolación de la Filosofía de Boecio. Estudia semejanzas entre el diálogo entre Celestina y Pármeno (auto 1) y del autor y de la Dama Filosofía en La consolación. Considera como ambas la Filosofía y Celestina pasan la vida tejiendo: una para unir con su labor la razón y la virtud, y la otra para conseguir el placer del hic et nunc.

685. GERLI, E. Michael. "Complicitous Laughter: Hilarity and Seduction in Celestina." Hispanic Review 16 (1995): 19-38.

Celestina es el primer texto que representa la risa como acto de significación. Hay variedades de la risa y se analizan aquí tres (Sempronio, Pármeno, Alisa). En cada instancia, la risa sirve para desenmascarar intenciones que desvirtúan el intento del discurso, y sirve un importante propósito artístico (permite ver subterfugios, contradicciones, etc.). El análisis del caso de Alisa es ingenioso.

686. GIMÉNEZ MICO, José Antonio. "Diversas conexiones entre Celestina y Elicia." Celestinesca 18.1 (primavera 1994): 35-50.

Se realzan en este estudio diferencias ideológicas y axiológicas entre LC y la obra del 'ciclo celestinesco,' Tragicomedia de Leandro y Roselia llamada Elicia, y por otro nombre cuarta obra y tercera Celestina (1542). Entre los temas comentados: el aburguesamiento de amo y criado, la institucionalización del discurso religioso y el comportamiento ideológico. Incluye un breve análisis del uso de referencias metatextuales en Elicia.

687. GONZÁLEZ DE LA RIVA LAMANA, J. M. "Los medicamentos en la literatura española" La Celestina" (V Congreso de la Organización Farmacéutica Ibero Latinoamericana). Alicante, 1992. $30 \mathrm{pp}$. 
Básicamente una lista de citas y explicaciones de los elementos del texto rojano a la luz de la decadencia médica, las supersticiones, etc. de la época de su confección. No cita fuentes de información y parece desconocer el libro de Laza Palacios (1958) y otros estudios anteriores sobre varios de estos elementos. Con todo, es informativo.

688. HATHAWAY, Robert. "Calisto and Melibea: Meeting Again for the First Time." Celestinesca 18.1 (primavera 1994): 87-91.

Tres recreaciones (verso y prosa) en distintos tonos inspiradas en la primera escena de Celestina.

689. HEUSCH, Charles. "La Célestine et la tradition amoureuse médiévale." Les Langues Neo-Latines, Núm 279 (1992): 5-24. (*)

690. HENRIQUEZ-SANGUINETI, Carolina. "La funcionalidad del grotesco en La Celestina y Jenofa Juncal de Alfonso Sastre," en Alfonso Sastre, ed. Mariano de Paco (Cuadernos, 60, Murcia: Universidad de Murcia, 1993), pp. 269-274.

Lo grotesco a servicio de la sátira y lo fantástico en un intento de desmitificar los valores españoles. En esta Celestina, la visión del hombre, de la mujer, de las instituciones, de la risallanto y del lenguaje producen un distanciamiento que nos lleva a reconocer que lo racional no forma parte de ningún cambio para mejor en cuanto a la situación satirizada.

691. HUERTA CALVO, Javier. "Tradición y modernidad en la novela sentimental: el ejemplo de Juan de Segura," en España, Teatro y Mujeres. Estudios dedicados a Henk Oostendorp, ed. Martin Grosman y Hub. Hermans. (Amsterdam/Atlanta: Rodopi, 1989), pp. 207-215.

Una demostración de cómo se puede ver en Proceso de cartas de amores de Juan de Segura la repetición de unos componentes tradicionales del género de la novela sentimental. Con ejemplos principales de Historia de duobus amantibus y la Comedia de Calixto y Melibea, el trabajo comenta el uso narrativo de la carta amorosa, además del lenguaje erótico y otros "elementos celestinescos." 
692. LARSEN, Kevin S. "Bed and Board: Significant Parallels Between Plato's Symposium and Rojas's La Celestina." Neohelicon 21 (1994): 247-268.

Amplia consideración de las posibles huellas de Platón en la Tragicomedia (especialmente del Simposio), siempre vistas por el lente de las transformaciones irónicas de Rojas. Bien interesante es la discusión de una Celestina modelada - en sentido inverso - en Sócrates.

693. LOLAS, Jazmín. "Un clásico para la pausa creativa." La Época (Santiago de Chile) (8 de mayo 1992), p. 24.

Opiniones de Alejandro Sieveking, director de la producción, sobre Celestina (obra y personaje).

694. MALACHY, Thérèse. "La Célestine de Fernando de Rojas: un drame à tiroirs." Revue d'Histoire du Théâtre, Núm 3 (1990): 223-241.

Pasa revista de tres temas: Celestina en su momento históricoliterario; Celestina vista a la luz bajtiniana; y una consideración breve de las recientes adaptaciones francesas (Achard, Laville, Vitez).

695. MENÉNDEZ PELAEZ, Jesús. "Entre la edad media y el renacimiento: La Celestina." Historia de la Literatura Española. I: Edad Media (León: Everest, 1993), pp. 465-483. Con ilustraciones.

Breve estudio liminar de la obra que bosqueja para el lector la historia textual temprana, teorías de la autoría y género (opta por su carácter dramático). Aboga por su postura moral.

696. MCGRADY, Donald. "Two studies on the text of the Celestina." Romance Philology 48 (1994): 1-21.

El primero de los estudios contiene interesantes comentarios al manuscrito de Palacio 1520 y su utilidad en la resolución de varios problemas sobre el comienzo del texto impreso; el segundo defiende las lecturas de las comedias - "Eras y Crato," "piedad de silencio," y "plebérico corazón" (el de 
Pleberio) -, creando una lectura que tiene buen sentido textual-literario.

697. MONGE, Félix. "El tipo celestinesco en la literatura clásica española" (discurso de ingreso). Zaragoza: Real Acad. de Bellas Artes de San Luis, 1993. 38 pp.

Pasa revista de una serie de manifestaciones celestinescas desde Ovidio hasta La Dorotea de Lope de Vega - y concentra en esta última obra, rememorando datos ya aducidos en sus estudios anteriores (de 1958, 1978, y 1983). Incluye algunas notas generales sobre Trotaconventos del Libro de buen amor.

698. MORON ARROYO, Ciriaco. Celestina and Castilian Humanism at the End of the Fifteenth Century. Occasional Papers, 3, Binghamton: Center for Medieval \& Early Renaissance Studies, 1994. $40 \mathrm{pp}$.

A la luz de su discusión de cristianos viejos y cristianos nuevos, el autor defiende su visión de un Rojas como moralista y humanista en los actos 2-16 de la Comedia (más que el autor del primer acto), y el concepto de amor como más caballaresco que cortés. Defiende además la validez de la etiqueta "comedia" en que las muertes ocurridas son productos de acciones individuales sin la participación de agente exterior alguno, y eso sería otra huella humanista.

699. MUÑOZ, H., Juan Antonio. "La Celestina o la ética del bolsillo." El Mercurio (Santiago de Chile) (8 de mayo 1992), p. 9 (suplemento). Con dos fotos.

Comentarios de adaptadores, director y actriz principal (Bélgica Castro) sobre Celestina (obra y personaje) en la ocasión de una nueva producción en Santiago ( 8 de mayo 1992).

700. NARVAEZ DE VELILlA, Francisco. Diálogo intitulado el Capón. Prólogo y edición de Víctor Infantes y Marcial Rubio Arquez. Madrid: Visor, 1993. Rústica, 123 pp. 
En el prólogo (9-44), passim, se reconoce la deuda con Celestina de esta obra dialogada.

701. OYARZUN L., Carola. "La Celestina." El Mercurio (Santiago de Chile) (5 de junio 1992), p. C19.

Reseña a la producción en Santiago dirigida por A. Sieveking (con Bélgica Castro en Celestina).

702. PASSALACQUA C., Italo. "Una 'Celestina' desnivelada." La Segunda (Santiago de Chile) (11 de mayo 1992), p. 35.

Una reseña a la producción de Santiago dirigido por Alejando Sieveking (con Bélgica Castro en Celestina).

703. PEREZ VIAL, María Pilar. Vigencia de una contemporánea Celestina. El Mercurio, Santiago (27 octubre 1991), p. E14.

La autora reproduce opiniones sobre la figura de Celestina del famoso ensayo (1938) de Ramiro de Maeztu.

704. PESTANO FARIN̄A, Rafael. "Pamphilus, de amore." Revista de Filología (Univ. de La Laguna) 6-7 (1987-1988): 357-365.

Pretende tomar aquí un primer paso - con un comentario analítico de las situaciones y caracterizaciones concretas del Pamphilus, de Amore - hacia una nueva valorización de los vínculos entre esta obra $-y$ su tradición $-y$ Celestina.

705. RAMOS OREA, Tomás. "Notas a otra lectura de La Celestina." El Correo de Andalucía (sección 'La Mirada,' 30 septiembre 1994), pp. 34-35. Ilustrada. También con una foto de la producción de la CNTC.

Una lectura-comentario de la obra que concentra en los diálogos amplios (los que hace al autor creer que LC es más novela que otra cosa). Ensayo de carácter personal.

706. RODRIGUEZ DIAZ, Rafael. "La Celestina, un original poema de amor," en 5 Estudios sobre literatura (San Salvador: UCA Editores, 1989), pp. 131-148. 
Comenta los muchos fracasos que llenan el texto celestinesco como una manera en la que Rojas plasma el drama humano de su grupo social (es decir, el fracaso de los conversos). La astucia, al servicio del intento de sobrevivir, no une a las personas sino que las separa. Este será el "mensaje" de la obra para el hondureño de hoy, para quien Celestina encapsula algunas de sus circunstancias históricas.

707. RODRIGUEZ TABOADELA, Nicanor. La Celestina, apoteósis de la fe católica y de la justicia. [Badajoz]: autor, 1987. 100 pp.

Ensayo extenso de carácter personal, repleto con citas textuales de LC, que aborda estos temas: Rojas, abogado altamente moral, casi teólogo; Rojas, único autor de una obra decididamente dramática; Sevilla como indudable lugar de la acción; la obra vista como demostración de la fe y enseñanzas católicas, y también de la presencia de una justicia divina. La bibliografía citada es pobre.

708. ROJAS, Fernando de. La Celestina. Adaptación de Miguel Reino. Colección 'Leer en español', Salamanca: Univ. de Salamanca/Santillana, 1994. Rústica, 120 pp. Portada ilustrada.

Adaptación ilustrada de Celestina (por Luis Jover) en cinco 'partes,' en algo menos de 100 páginas. Acompañada de preguntas para ayudar a los jóvenes a comprender la obra, con una serie de notas útiles.

709. La Celestina. Edición, notas y actividades de Manuel Cifo González. Col. Anaquel, 29, [Madrid]: Bruño, 1993. Rústica, 379 pp. Cubierta Ilus. de Javier Zabala.

Texto con ilustraciones. Edición estudiantil con notas léxicas, una mínima introducción (6 pp.), una cronología (6 pp.), un "análisis" de la obra (21-46) adecuado sólo para los alumnos, una bibliografía comentada con sólo 10 entradas (!), y el texto completo de la TCM. Seguido por unas útiles actividades para profundizar en el texto (349-379). Incluye unos índices analíticos: temas, referencias y citas, y arcaísmos. . La Celestina. CEE: PML Ediciones, 1994. Rústica 254 pp. 
El texto completo de la TCM, con una introd. anónima de 3 páginas. Sin notas, bibliografía, ilustraciones.

711. La Celestina. Two Facsimiles (1499? and 1528). New York: The Hispanic Society of America, 1995. n.p.

El texto facsímil de Burgos?, 1499? y Sevilla 1528, sin introducción $u$ otro aparato crítico. Ambas ediciones ilustradas.

712. SALAZAR Y TORRES, Agustín de., Juan de Vera Tassis y Villarroel, y Sor Juana Inés de la Cruz. El encanto es la hermosura y el hechizo sin hechizo / La segunda Celestina. Edición, introducción y notas de Thomas Austin O'Connor. Binghamton: Pegasus Paperbooks, 1994. Rústica, 180 pp.

Celestina en manos de Salazar y Torres y Vera Tassis pasa por una doble trayectoria dramática: la mitificación y la desmitificación (Intro., xxviii-xxxv). La figura de la alcahueta se debilita en la final de Sor Juana.

713. SAMONA, Carmelo. "La nascita del teatro moderno: la città, $\mathrm{i}$ pastori, la corte," en La letteratura spagnola dal Cid ai Re Cattolici (Firenzi: Sansoni, 1972): 215-251).

Una serie de ensayos sobre las circunstancias históricoliterarias de la aparición de Celestina, su autor, y su arte (esp. la retórica). Dedica sus prịmeros comentarios - como pocos críticos hacen ahora - a la versión en 16 autos, y luego la de 21 actos, y demuestra que cada una tiene una unidad de composición, y de estilo notable. Explora la importancia de la causalidad como fuerza motriz de la unicidad de la obra de Rojas; elogia los logros que ostenta la vida urbana (nueva) retratada en ella. Defiende, en las páginas finales (243-47) los aciertos de ocho interpolados.

714. SANZ HERMIDA, Jacobo. "Una vieja barbuda que se dice Celestina: Notas acerca de la primera caracterización de Celestina." Celestinesca 18.1 (primavera 1994): 17-33.

Sugiere que la primera caracterización de Celestina - como 'vieja barbuda' - no es cuestión de un mero epíteto, sino que 
refleja la personalidad compleja de este personaje según una larga tradición literaria. Al analizar el vasto campo de conotaciones con respecto a las barbudas, descubrimos que el calificativo que Sempronio emplea no es utilizado por casualidad.

715. SCOLES, Emma. "Risonanzia della Celestina in un testo teatrale portoghese del XVIII secolo," en De una riva e dall'altra. Studi in onore di Antonio D'Andrea, a cura di Dante Della Terza (Firenze: Ed. Cadmo, 1994). (*)

716. SNOW, Joseph T. "Celestina de Fernando de Rojas: documento bibliográfico (décimosepto suplemento)." Celestinesca 18.1 (primavera 1994): 93-117.

Ampliación de su bibliografía celestinesca (1985) con 106 entradas nuevas.

717. SWAFFORD-SMITH, Christine. "The Aesthetics of Death: Buen Amor, 'Coplas por la muerte de su padre,' and the Celestina." Disertación doctoral, Indiana Univ., 1989. vii + 293 pp. DAI, Ann Arbor MI, núm. de pedido \#8925171.

El cuarto capítulo (252-293) contiene el estudio de Celestina. Se distinguen dos perspectivas sobre la muerte: (1) la tendencia a usarla para referirse a tres aspectos de la vida (los sentidos descriptivo, persuasivo y catártico) y, (2) a partir de la entrada física de la muerte (final del auto XII) como poder destructivo de la dinámica de la vida y de los procesos de renovación y como anticipo del caos final. La muerte es, de una mano, una parte importante de la vida, sin participar o interesarse, de la otra mano, en ella.

718. TRONCONE, Carlos. "Demasiado humana." Teatro 2 (Buenos Aires) 4, no. 5 (abril 1994):.62-65.

Es un reportaje sobre la obra y su historia (con errores de algún dato) a raíz de una nueva producción de Celestina en Buenos Aires. Incorpora entrevistas a Osvaldo Bonet (director) y Graciela Araujo (actriz que hace Celestina). Ilustrado con fotos de la producción y de los entrevistados. 
719. VÉLEZ QUIÑONES, Harry. "Cruel and Pathetic Dissonance: The Grotesque and the Celestinas." Revista de Estudios Hispánicos 24.1 (enero 1995): 51-72.

Celestina, en los momentos en que lo familiar se vuelve ajeno, crea la pauta de lo grotesco para otros textos posteriores (notablemente la Tercera Celestina de Gómez de Toledo). Esta cohabitación de lo más alto con lo más bajo, que genera las disonancias (el autor analiza la figura de Celestina del primer auto, su muerte, y las siguientes muertes con grandes aciertos), parece estar ausente en la Segunda Celestina de Feliciano de Silva, pero luego restaurado con brillantez por Gómez de Toledo, tal vez el mejor exponente de este tipo del grotesco.

720. La celestinesca, la comedia y 'La Dorotea': Huellas de un intertexto. Col. Aquilafuente, 5, Salamanca: Ediciones Universidad, 1994. 10 pp. +1 microficha.

Una reproducción, esencialmente, de la tesis doctoral leída en Harvard, en la que explora en intertexto que vinculan las 'celestinas' (La comedia Tebaida, La Segunda Celestina, La Tercera Celestina, Lisandro y Roselia, Tragedia Policiana, Florinea, Selvagia, Comedia Euphrosina) a la obra de Rojas y, al mismo tiempo, unas con otras, acabando con textos de Lope (El rufián Castrucho, El caballero de Olmedo, La Dorotea), consideradas todas ellas a la luz interpretativa del imitatio.

721. VILLEGAS, Juan. "Humor, sexualidad y desengaño en la Celestina de Alfonso Sastre." Anthropos 126 (nov. 1991): 57-59.

Sastre introduce en Celestina la nota cómica, popular. Importancia de Sempronio como portavoz en la obra. Calisto y Melibea condicionados por la debilidad del cuerpo.

722. . "La Celestina de Alfonso Sastre: Niveles de intertextualidad y lector potencial," en Alfonso Sastre, ed. M. de Paco (Cuadernos 60, Murcia: Univ. de Murcia, 1993), pp. 265-268. Reimpresión del estudio aparecido en Estreno 12.1 (1986): 4041. 